Check for updates

Cite this: RSC Adv., 2018, 8, 14009

\title{
Effects of biopolyimide molecular design on their silica hybrids thermo-mechanical, optical and electrical properties $\uparrow$
}

\begin{abstract}
S. Dwivedi, (D) ${ }^{a}$ S. Sakamoto, ${ }^{a}$ S. Kato, ${ }^{c}$ T. Mitsumata ${ }^{\text {bc }}$ and T. Kaneko*ab
Polymers, derived from bio-derived resources, have gained considerable momentum because of a lower dependence over conventional fossil-based resources without compromising the materials' thermomechanical properties. Unique characteristics of organic and inorganic materials can be incorporated by a combination of both to obtain hybrid materials. We have recently developed a series of transparent biopolyimides (BPI) from a biologically derived exotic amino acid, 4-aminocinnamic acid (4ACA) to yield 4-amino truxillic ester (4ATA ester) derivatives. In the present research, the polyimide-precursor was subjected to sol-gel polycondensation reactions with silicon-alkoxide followed by annealing under vacuo to yield a biopolyimide-silica hybrid. The biopolyimide structures (4ATA acid/ester) and their silica hybrids thermo-mechanical, electrical and optical performance were evaluated. It was found that the biopolyimide with 4ATA(ester) yields thermo-mechanically robust films with very high electrical stability as well as optical transparency, plausibly due to the uniform dispersion of the silica particles in the biopolyimide matrix.
\end{abstract}

Received 6th March 2018

Accepted 3rd April 2018

DOI: $10.1039 / \mathrm{c} 8 \mathrm{ra0} 01965 \mathrm{~g}$

rsc.li/rsc-advances
High-performance polymers are used in applications demanding service at enhanced temperatures, while maintaining their structural integrity and an excellent combination of chemical, physical, and mechanical properties. ${ }^{15}$ Among them, aromatic polyimides represent an increasing important class of materials in aerospace, microelectronics, and other industrial applications. ${ }^{\mathbf{1 6 - 1 9}}$ Polyimides are one of the important classes of polymers used as interlayer dielectrics for advanced printed circuit boards and multichip module packing. Dielectric materials used for thin film multichip modules must meet a number of material and electrical requirements including a low-dielectric constant, low-dissipation factor, minimal moisture absorption, and high thermal stability. ${ }^{\mathbf{2 0 - 2 3}}$ Many desirable properties of polyimides, including good thermo-oxidative stability and excellent mechanical properties, contribute to their success. However, the polyimides exhibit relatively high dielectric constant. A way to obtain materials with improved properties is to form organicinorganic hybrids by incorporating inorganic fillers. The hybrid polyimides represent a class of new generation of materials that combine the properties of the ceramic phase with those of organic polymers. ${ }^{24}$ The incorporation of various metallic additives, such as $\mathrm{TiO}_{2}, \mathrm{BaTiO}_{3}, \mathrm{Al}_{2} \mathrm{O}_{3}, \mathrm{SiO}_{2}$ and, $\mathrm{ZnO}$ into polyimides has been reported to improve the properties of the resulted materials. ${ }^{25-27}$ However, the importance of polyimide microstructure and the performance of their hybrids have been rarely systematically studied.

The sol-gel technique is an excellent method to produce hybrid polyimide/silica polymers. This method consists hydrolysis of an alkoxysilane and a polycondensation process. ${ }^{28,29}$ Therefore, the 
processing of the polyimides is generally carried out via soluble poly(amic acid) precursors, which are cast onto various substrates, and then they are converted into polyimide films by thermal treatment. The good solubility of the poly(amic acid) precursors in amidic solvents makes possible the introduction of metal oxide precursors into their solutions. Also, the excellent thermal stability of polyimides makes possible the required thermal treatment up to high temperatures without inducing appreciable degradation in the organic phase. Lee et al., found that the phase separation occurred at higher silica content leads to the lower mechanical strength of the resulting film relative to the pure polyimide or lower silica content containing polyimide. ${ }^{30,31}$ Therefore, the compatibility between the polyimide and silica should be improved by precise monomer molecular design.

Recently, we have established the syntheses of a diamine from biologically obtained exotic amino acid, 4-aminocinnamic acid (4ACA) to yield 4,4'-diamino truxillic ester (4ATA ester), used for the biopolyimide preparation. ${ }^{32,33}$ In this article, we report the preparation of a new diamine $4,4^{\prime}$-diamino truxillic acid and a series of biopolyimide and their silica hybrids. The affinity of silica phase with the polymeric matrix, was controlled with the acid/ester functionalization of the diamine moiety in the biopolyimide. The biopolyimide structural characteristics and the thermo-mechanical, electrical and the optical performances were correlated.

\section{Experimental}

\section{Materials}

Dianhydride, 1,2,3,4-tetracarboxycyclobutane dianhydride (CBDA, Tokyo Chemical Industries (TCI), Tokyo, Japan) was purified by sublimation under reduced pressure before use. Diamine precursor 4-aminocinnamic acid (4ACA, TCI, Tokyo, Japan), esterification reagent trimethylsilyl chloride (TMSCl, SigmaAldrich, Tokyo, Japan), and polymerization solvent superdehydrated N,N-dimethylacetamide (DMAc, Kanto Chemical
Corporation, Tokyo, Japan) and solvents were used as received. All chemicals used were of research grade.

\section{Syntheses}

Monomers were synthesized with the Scheme S1. $\dagger$ 4,4'-Diamino- $\alpha$-truxillic acid (4ATA) dihydrochloride was synthesized by the drop-wise addition of $12 \mathrm{~N}$ hydrochloric acid solution (5.6 $\mathrm{ml})$ in the solution of 4ACA $(2.0 \mathrm{~g}, 12.4 \mathrm{mmol})$ in acetone $(30 \mathrm{ml})$ to produce 4-aminocinnamic acid hydrochloride $(1.74 \mathrm{~g}, 4.35$ $\mathrm{mmol}$ ). The obtained product was subjected to irradiation by a $100 \mathrm{~W}$ high pressure Hg-lamp (Omni Cure S1000, EXFO Photonic Solution Inc.) with a $250-450 \mathrm{~nm}$ band-pass filter with an intensity of $2.7 \mathrm{~mW} \mathrm{~cm} \mathrm{~cm}^{-3}$ for $24-36 \mathrm{~h}$ to induce $[2+2]$ photocycloaddition. The reaction was monitored by ${ }^{1} \mathrm{H}$ NMR (400 MHz, DMSO- $\mathrm{d}_{6}, \delta, \mathrm{ppm}$ ) by disappearance of peak for the olefinic protons: $3.82(\mathrm{dd}, 2 \mathrm{H}, J=7.7,9.6 \mathrm{~Hz}), 4.30(\mathrm{dd}, 2 \mathrm{H}, J=$ 7.7, $9.6 \mathrm{~Hz}), 7.33$ (d, $4 \mathrm{H}, J=7.7 \mathrm{~Hz}), 7.45$ (d, $4 \mathrm{H}, J=7.7 \mathrm{~Hz}$ ), 10.37 (s, 6H), 12.07 (s, 2H).

The obtained 4,4'-diamino- $\alpha$-truxillic acid (4ATA) dihydrochloride was subjected to the esterification. A solution of 4 ATA $(1.71 \mathrm{~g}, 4.27 \mathrm{mmol})$ in methanol was prepared and TMSCl was added drop-wise. The reaction was monitored by ${ }^{1} \mathrm{H}$ NMR (400 MHz, DMSO- $\mathrm{d}_{6}, \delta, \mathrm{ppm}$ ) and allowed to takes place $12 \mathrm{~h}$. The obtained product was filtered and dried at $40{ }^{\circ} \mathrm{C}$ under vacuo for $6 \mathrm{~h}$. The dimethyl ester of 4ATA salt $(1.83 \mathrm{~g}, 4.28 \mathrm{mmol})$ was dissolved in water and neutralized by $1 \mathrm{~N} \mathrm{NaOH}$ solution to obtain 4,4'-diamino- $\alpha$-truxillic dimethyl ester (1.29 g, 3.64 $\mathrm{mmol})$. The obtained product was dried and subjected to soxhlet purification using ethyl acetate to get crystals. The purified 4,4'-diamino- $\alpha$-truxillic dimethyl ester was confirmed using ${ }^{1} \mathrm{H}$ NMR (400 MHz, DMSO-d $\mathrm{d}_{6}, \delta$, ppm).

The crystals of $4,4^{\prime}$-diamino- $\alpha$-truxillic dimethyl ester, $(0.20 \mathrm{~g}, 0.5647 \mathrm{mmol})$ was dissolved in DMAc $(0.8 \mathrm{ml}, 9.6 \mathrm{mmol})$ under a nitrogen atmosphere followed by the addition of CBDA $(0.11 \mathrm{~g}, 0.5647 \mathrm{mmol})$, as shown in the Scheme 1 . The reaction mixture was stirred at room temperature to produce a clear to

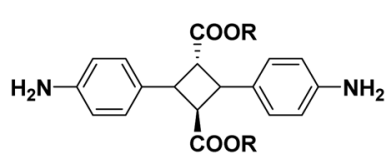

4,4'-Diamino- $\alpha$-truxillic dimethyl acid/ester

$\mathrm{R}=\mathrm{CH}_{3}, \mathrm{H}$

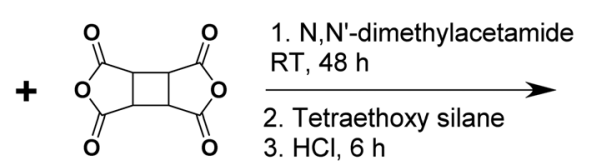

Dianhydride

\footnotetext{
- Thermal stability

- Mechanical strength

- Optical transparency

- Dielectric properties

- Breakdown voltage
}
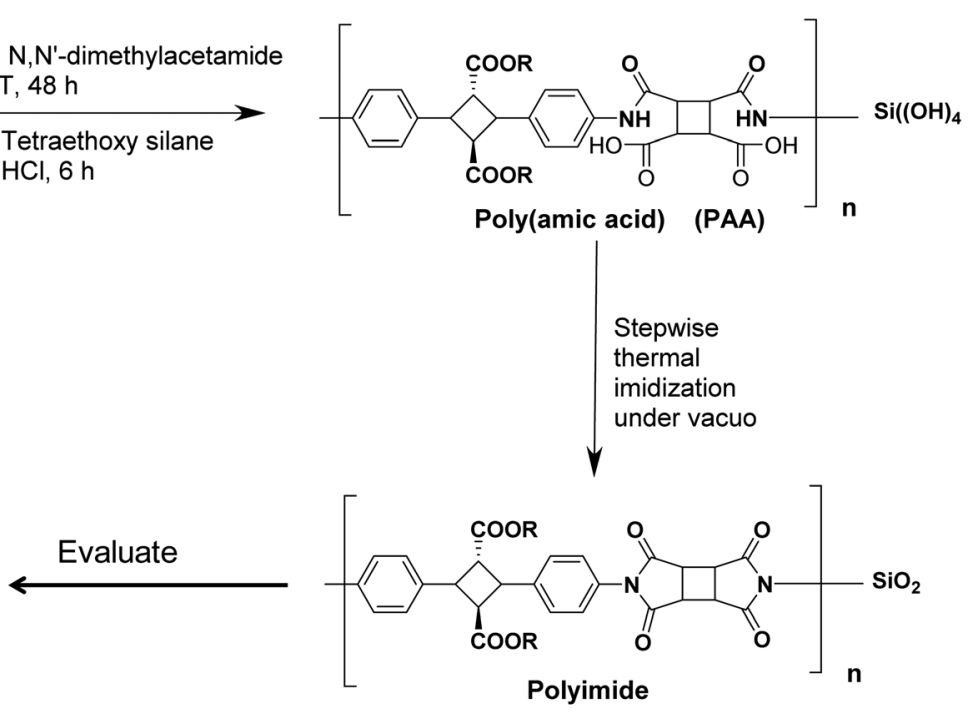

Scheme 1 Polymerization reaction scheme and the resulting biopolyimide/silica hybrids evaluated properties. 
pale yellow viscous poly(amic acid) (PAA) solution in $48 \mathrm{~h}$. The PAA solution was diluted in DMAc, and then added dropwise in methanol/water mixture to precipitate PAA fibers, which were filtered and dried under vacuo at $60{ }^{\circ} \mathrm{C}$ for $12 \mathrm{~h}$. The PAA film was obtained by casting a PAA homogeneous solution in DMAc onto a glass plate and heating at $75{ }^{\circ} \mathrm{C}$. The obtained PAA films were subjected to a thermal imidization in an oven under reduced pressure by stepwise heating at 100,150 and $200{ }^{\circ} \mathrm{C}$ for $1 \mathrm{~h}, 1 \mathrm{~h}$ and $3 \mathrm{~h}$ at each step respectively to obtain the polyimide films. Similar procedure for the polyimide preparation has been followed with other dianhydride structures.

Biopolyimide-silica hybrids were synthesized by the sol-gel condensation. Pre-calculated amount of TEOS was added in the poly(amic acid) solution in the DMAc followed by the addition of $\mathrm{HCl}$ (catalyst) to yield the silica formation the polymer matrix. Gradual annealing of the resulting poly(amic acid)/silica films up to $200{ }^{\circ} \mathrm{C}$ results the formation of the biopolyimide-silica hybrids.

\section{Characterization}

The number-average molecular weight $\left(M_{\mathrm{n}}\right)$, weight-average molecular weight $\left(M_{\mathrm{w}}\right)$ and the molecular weight distribution (PDI) were determined by gel permeation chromatography (GPC, concentration $5 \mathrm{~g} \mathrm{~L}^{-1}$, DMF eluent, Shodex SB-800HQ, Showa Denko K.K., Tokyo, Japan) after calibration with pullulan standards.

NMR measurements were performed by a Bruker Biospin AG $400 \mathrm{MHz}, 54 \mathrm{~mm}$ spectrometer using DMSO- $_{6}$ as the solvent. The FT-IR spectra were recorded with a Perkin-Elmer Spectrum One spectrometer between 4000 and $600 \mathrm{~cm}^{-1}$.

Thermogravimetric analysis (TGA) was performed on STA 7200 (Hitachi, Tokyo, Japan) at a heating rate of $10{ }^{\circ} \mathrm{C} \mathrm{min}-1$ under a nitrogen atmosphere. The polymer specimens were dried at $100{ }^{\circ} \mathrm{C}$ for $1 \mathrm{~h}$ to remove any absorbed moisture before both TGA. The tensile measurements were carried out at an elongation speed of $0.5 \mathrm{~mm} \mathrm{~min}^{-1}$ on a tensiometer, the Instron 3365 at room temperature.

The ultraviolet-visible (UV-vis) optical absorption spectra were recorded on a Perkin-Elmer, Lambda $25 \mathrm{UV} / \mathrm{vis}$ spectrophotometer at room temperature over the range of 200-800 $\mathrm{nm}$. X-ray diffraction (XRD, Rigaku SmartLab, Tokyo, Japan) pattern was used to determine the crystallinity degree of the polyimide films. The morphology and the elemental composition of the polyimide films were determined by scanning electron microscope (SEM, JEOL Japan).

The dielectric constant for the biopolyimide (BPI) and their silica hybrids was measured by using a LCR meter (IM 3536 HIOKI, Nagano, Japan) at $293 \mathrm{~K}$. An electric voltage of $1.0 \mathrm{~V}$ was applied by two electrodes made up of brass. The frequency was kept at $1 \mathrm{MHz}$.

The dielectric breakdown voltage was measured by using an electrical safety analyzer (SE7430 Keisoku Giken, Yokohama, Japan) at room temperature. The electric voltage was applied by two terminals method up to $6 \mathrm{kV}$ for DC with a ramp-up time of $120 \mathrm{~s}$ and with a rate of $1 \mathrm{kV} \mathrm{s}^{-1}$. The electrode was made of stainless steel with a diameter of $20 \mathrm{~mm}$. In the process, the samples were cut to dimensions of $40 \times 40 \mathrm{~mm}$. The electric breakdown (EBD) strength of the films was calculated by dividing the electric breakdown voltage by the thickness of samples.

\section{Results and discussion}

\section{Material syntheses}

Production of diamine from 4ACA demands the amalgamation of two precursor units. A [2+2] photocycloaddition reaction of trans-cinnamic acid, with radiations $(\lambda>260 \mathrm{~nm})$ to yield $\alpha$ truxillic acid. Olefinic functionality of 4ACA was utilized to obtain the corresponding $\alpha$-truxillic acid from the 4ACA salt, characterized by ${ }^{1} \mathrm{H}$ NMR (Fig. S1 $\dagger$ ). The reaction was monitored by the ${ }^{1} \mathrm{H}$ NMR with the disappearance of the olefinic protons (7.44-7.40 and 6.15-6. 12) and development of cyclobutane proton signals (4.36-3.80) (Fig. S2 $\dagger$ ). Thereafter, $\alpha$-truxillic acid salt was neutralized by $1 \mathrm{M} \mathrm{NaOH}$ and purified by activated charcoal to obtain 4ATA (acid type, Fig. S3†). On the other hand, as precise monomer design demands the protection of the twocarboxylic acid groups of 4ATA to avoid any possible interference during the polymerization. Therefore, esterification of 4ATA was performed with methanol and TMSCl, confirmed by the ${ }^{1} \mathrm{H}$ NMR (Fig. S4 $\dagger$ ). Finally, the 4ATA-methyl ester salt was neutralized by $1 \mathrm{M} \mathrm{NaOH}$ aqueous solution and the final product was confirmed by ${ }^{1} \mathrm{H}$ NMR (Fig. S5 $\dagger$ ).

The polycondensation was carried out in a $1: 1$ solution of 4ATA ester/acid and dianhydride in the super dehydrated DMAc. ${ }^{21}$ The development of viscosity with the progression of the reaction indicates the formation of poly(amic acid) (PAA). FT-IR analysis of the PAA fibrils shows a broad signal around $2600-3600 \mathrm{~cm}^{-1}$ (O-H, stretching), two sharp absorption bands at $1720 \mathrm{~cm}^{-1}$ (C-O stretching, carboxylic and ester) and $1670 \mathrm{~cm}^{-1}$ (C-O stretching, amide) and aromatic peaks at $1525 \mathrm{~cm}^{-1}$ and $1432 \mathrm{~cm}^{-1}$ (C-H first overtone, aromatic) (Fig. S6 $\dagger$ ). TEOS with catalyst $(\mathrm{HCl})$ lead to the sol-gel condensation lead to the silica precursor formation. The number average molecular weight $\left(M_{\mathrm{n}}\right)$ and PDI of the PAA was measured by GPC, which were found to be $4.7-5.2 \times 10^{5}$ and 1.4 respectively. PAA films were prepared by solution casting and stepwise annealing yields polyimide films. The chemical structure of the PI was confirmed by the FT-IR. A signal at $1375 \mathrm{~cm}^{-1}$ and $1175 \mathrm{~cm}^{-1}$ (C-N stretching, imide) confirms the formation of imide ring and the imidization under annealing process. The peak at $1211 \mathrm{~cm}^{-1}$ has been assigned mainly to the $\mathrm{C}-\mathrm{O}$ (stretch, $\mathrm{COOH}, \mathrm{COOCH}_{3}$ ). Furthermore, the spectra shows two peaks for the carbonyl at $1785 \mathrm{~cm}^{-1}(\mathrm{C}=\mathrm{O}$, asymmetric stretching) and $1716 \mathrm{~cm}^{-1}(\mathrm{C}=\mathrm{O}$ symmetric stretching). The obtained polyimide was confirmed to have high chemical resistance except for concentrated sulfuric acid.

\section{Optical properties}

The neat biopolyimide (BPI) and their silica hybrid films were prepared and subjected to the UV-vis spectroscopy for understanding their optical properties. It was interesting to observe that the $\mathrm{COOCH}_{3}$ type BPI were more transparent than $\mathrm{COOH}$ 
Table 1 Thermo-mechanical and optical properties of biopolyimide and their silica hybrids

\begin{tabular}{|c|c|c|c|c|c|c|c|}
\hline Polyimide type & $\mathrm{SiO}_{2}$ content (wt $\left.\%\right)$ & $\delta^{a}(\mathrm{MPa})$ & $\varepsilon^{a}(\%)$ & $E^{a}(\mathrm{GPa})$ & $T_{\mathrm{d} 1}^{b}\left({ }^{\circ} \mathrm{C}\right)$ & $T_{\mathrm{d} 10}^{b}\left({ }^{\circ} \mathrm{C}\right)$ & $T_{450 \mathrm{~nm}^{c}}(\%)$ \\
\hline \multirow[t]{3}{*}{$\mathrm{COOH}$} & 5 & 110 & 3.9 & 4.1 & 326 & 403 & 85.7 \\
\hline & 10 & 122 & 5.5 & 6.6 & 332 & 397 & 77.1 \\
\hline & 20 & 119 & 6.2 & 4.6 & 204 & 249 & 73.9 \\
\hline \multirow[t]{2}{*}{$\mathrm{COOCH}_{3}$} & 5 & 84 & 3.0 & 2.7 & 319 & 377 & 86.6 \\
\hline & 10 & 112 & 7.0 & 3.5 & 323 & 381 & 88.3 \\
\hline
\end{tabular}

${ }^{a} \delta, \varepsilon$ and $E$ refers to tensile strength, elongation degree and modulus of elasticity respective determined by the tensile tests. ${ }^{b} T_{\mathrm{d} 1}$ and $T_{\mathrm{d} 10}$ represents the $1 \mathrm{wt} \%$ and $10 \mathrm{wt} \%$ thermal degradation temperature, respectively, measured under nitrogen through TGA. ${ }^{c} T_{450} \mathrm{~nm}$ shows the transmittance at $\lambda=450 \mathrm{~nm}$ was measured by UV-vis spectroscopy.

type BPI. However, this gap in the transparency gets wider with the incorporation of silica in the BPI. The $\mathrm{COOCH}_{3}$ type BPI silica hybrids transparency improved with the silica incorporation. On the contrary, in the case of the $\mathrm{COOH}$ type BPI the transparency was reduced with silica incorporation, which may be attributes to the larger silica formation in the polymer matrix, responsible for the light reflection/scattering rather than transmission. In order to confirm the silica particle distribution homogeneity in the polymer matrix, SEM analyses was conducted over the film surface. It was observed that in the case of $\mathrm{COOH}$ type BPI, silica particles were present in the form of micro-meter sized particles. On the contrary, $\mathrm{COOCH}_{3}$ type BPI silica hybrids show rather more uniform surface. Thereby, we confirmed the silica particle formation in the COOH type BPI was more non-uniform than $\mathrm{COOCH}_{3}$ type BPI (except at over loading ca. $20 \mathrm{wt} \%$ ). The silica particle dispersion was confirmed using the SEM analyses.

\section{Thermo-mechanical properties}

Thermal stability of the BPI and their silica hybrids were studied by thermogravimetry, by heating sample from $25^{\circ} \mathrm{C}$ to $800{ }^{\circ} \mathrm{C}$ at the rate of $5{ }^{\circ} \mathrm{C} \mathrm{min}^{-1}$ under nitrogen. The degradation temperature of a material may be represented at various extent of material degradation. $\mathrm{Mu}$ et al., has shown the importance of $T_{\mathrm{d} 1}$ ( $1 \mathrm{wt} \%$ loss temperature) over other degradation temperatures. ${ }^{34}$ Considering the complexities of the thermal degradation, $T_{\mathrm{d} 1}$ and $T_{\mathrm{d} 10}$ (conventional degradation parameter in polymers) both have been mentioned in the Table 1 . It was interesting to observe that both the $T_{\mathrm{d} 1}$ and $T_{\mathrm{d} 10}$ follows almost same trend for the BPI silica hybrids. It was observed that the $\mathrm{COOH}$ type BPI was more thermally stable than the $\mathrm{COOCH}_{3}$ type BPI at $5 \mathrm{wt} \%$ of silica loading. The greater stability of the $\mathrm{COOH}$ type BPI may be envisaged to the possibility of $\mathrm{H}$-bonding interactions in the polymer matrix between $\mathrm{COOH}$ and other electron rich moieties $(\mathrm{O}, \mathrm{N})$ apart from the conventional charge-transfer (CT) interactions. Incorporation of silica in the BPI matrix leads to the decrease in the thermal stability for the COOH type. While, the $\mathrm{COOCH}_{3}$ type BPI silica hybrids thermal stability improved with the addition of silica, until $10 \mathrm{wt} \%$ and lead to a decrement in stability at $20 \mathrm{wt} \%$. The observation may be attributed to the enhanced uniform synergistic chain-transfer interactions between the silica units and the $\mathrm{COOCH}_{3}$ BPI chains. The non-uniformity (or over-loaded) in the silica distribution leads to the weaker synergism for CT interactions between silica and $\mathrm{COOH}$ type BPI.

The BPI silica hybrids robustness was judged through the mechanical testing (Table 1). It was observed that the $\mathrm{COOCH}_{3}$ type BPI silica hybrids shows an increase in modulus of elasticity, degree of elongation and the tensile strength with the silica content in the polymer matrix. It was interesting to note that the tensile strength of the $\mathrm{COOCH}_{3}$ type BPI silica hybrid was increased by over $77 \%$ than the neat BPI. While the modulus of elasticity was increased over $107 \%$ and degree of elongation by $86 \%$ for $20 \mathrm{wt} \%$ of silica incorporation in the $\mathrm{COOCH}_{3}$ type BPI structure. On the contrary, in the case of $\mathrm{COOH}$ type BPI, it was observed that the mechanical properties increased but with a relatively random trend. It was observed that the tensile strength of the BPI increased by around only 9\%. Moreover, modulus of elasticity and elongation degree improved by hardly $11 \%$ and $61 \%$ respectively. The higher tensile strength of the neat $\mathrm{COOH}$ BPI may be attributed to the more ordered structure of the BPI due to H-bonding in the polymer matrix. However, the slower increase in the $\mathrm{COOH}$ type PI silica hybrids mechanical properties can be a trait arose due to the disturbed H-bonding and the non-homogeneous silica particle distribution, which causes over stress along the particle grain boundary lead to easier film rupture. On the contrary, in the case of $\mathrm{COOCH}_{3}$ type BPI, the silica particles were more uniformly distributed (confirmed by SEM image, Fig. 1) along the polymer matrix and the stress was evenly shared along the

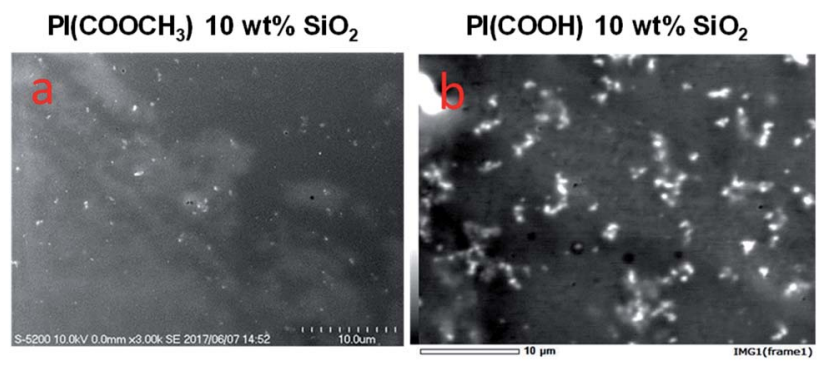

Fig. 1 Biopolyimide/silica SEM micrographs at 10 wt\% loading. 
matrix, which led to higher mechanical robustness development.

Furthermore, the biopolyimide crystallinity was investigated by X-ray diffraction (XRD). The XRD pattern of biopolyimide yields two diffraction peaks at $2 \theta=6^{\circ}$ and $17^{\circ}$ overlapping with a broad amorphous halo (Fig. S7†). The XRD diagram of neat biopolyimide indicates a partial crystallization (14-22\%). It is noteworthy to mention that the biopolyimide silica hybrids crystallinity decreased with the increase in the silica content, which may be envisaged as a reason for the improved elasticity of the biopolyimide-silica composites.

\section{Dielectric constant}

Materials with low dielectric constants (low- $\varepsilon$ ) are required by the modern integrated circuits (IC) in order to reduce the resistance capacitance (RC) delay and minimize cross-talk noise. In this respect, polyimide (PI) is desirable because of its high thermal stability, excellent mechanical properties, and low dielectric constant. ${ }^{35-38}$ Therefore, recent researches are focused on reducing the dielectric constant of PI by synthesis of novel structures and organic-inorganic composites, especially using sol-gel methodology ${ }^{36}$ Introduction of fillers into the PI matrix also can reduce $\varepsilon$ considerably. For example, Zhang et al. and Kim et al. incorporated silica tubes into PI by self-assembly method to obtain a composite with a low value of $\varepsilon$ (dielectric constant) up to 3.6-4.0.39,40 However, in spite of several attempts, the dielectric constant of PI composites is usually more than 3.5 which does not meet the stringent demand by the modern microelectronics industry. ${ }^{41-44}$

Fig. 2 shows the silica concentration dependence of dielectric constant for BPI hybrids. Dielectric constants of the BPI/ silica hybrids decreased from 4.6 (neat $\mathrm{COOH}$ type BPI) to 3.3 (50 wt\% silica). Neat $\mathrm{COOCH}_{3}$ type BPI possesses greater dielectric constant than $\mathrm{COOH}$ type BPI, which may be attributed to the inherent polarization of $\mathrm{COOCH}_{3}$ moiety. However, irrespective of kind of BPI, the dielectric constant almost

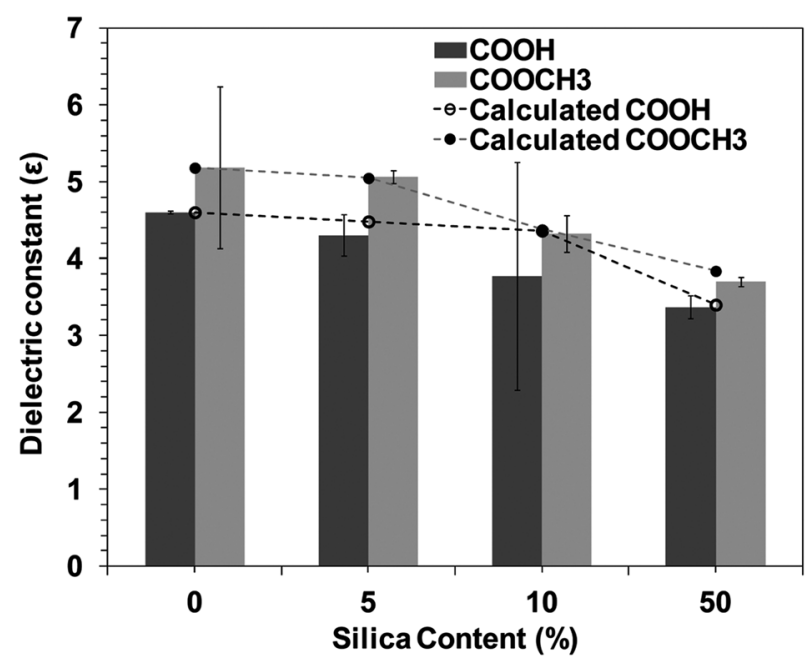

Fig. 2 Dielectric constant variations with silica content in biopolyimide. linearly decreased with the increase in the silica loading, suggesting that the decrease in the dielectric constant is due to the low dielectric constant of silica. The dielectric constant can be explained by the following equation, which is a linear combination of dielectric constant of BPI and silica,

$$
\varepsilon_{\text {hybrid }}=\varepsilon_{\mathrm{Si}} \varphi_{\mathrm{Si}}+\left(1-\varphi_{\mathrm{Si}}\right) \varepsilon_{\mathrm{BPI}}
$$

where, $\varepsilon_{\text {hybrid }}, \varepsilon_{\mathrm{Si}}$ and $\varepsilon_{\mathrm{BPI}}$ represents the dielectric constant for $\mathrm{BPI} /$ silica hybrid, silica, and biopolyimide respectively; $\varphi_{\mathrm{Si}}$ is the amount of silica. The line along the data in Fig. 2 represents the result of fitting by eqn (1). The dielectric constant for both biopolyimides was well fitted by eqn (1), suggesting that the interfacial polarization effect is less in the hybrids. The values of $\varepsilon_{\mathrm{Si}}$ were determined to be 2.6 for $\mathrm{COOH}$ and 3.5 for $\mathrm{COOCH}_{3}$, which were close to the literature values of silica. ${ }^{45}$

\section{Voltage endurance}

Moreover, the demand for improved energy storage solutions has driven the development of power conditioning and management devices that endure higher operating voltages with greater reliability. The maximum energy an electrostatic capacitor can store is dependent on the dielectric permittivity and breakdown voltage of the insulating material separating the electrodes. ${ }^{46-50}$ For instance, commonly used polymer dielectric BOPP (biaxially oriented polypropylene) has relatively high

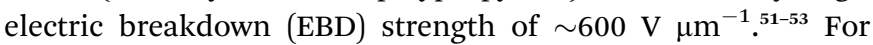
electronic grade amorphous polymers, high breakdown strength has been previously associated with polar polymers.

The DC breakdown voltages of the BPI films with/without silica were measured. The BPI film thickness with and without silica were kept almost uniform, $27 \pm 5 \mu \mathrm{m}$ (Fig. S8†). Fig. 3 shows the silica concentration dependence of the DC breakdown voltage for BPI hybrids. It was observed that the introduction of the silica in the BPI matrix increases the DC breakdown voltage. However, the increase in the DC breakdown voltage was gradual in the case of $\mathrm{COOH}$ type BPI, i.e. up to 108

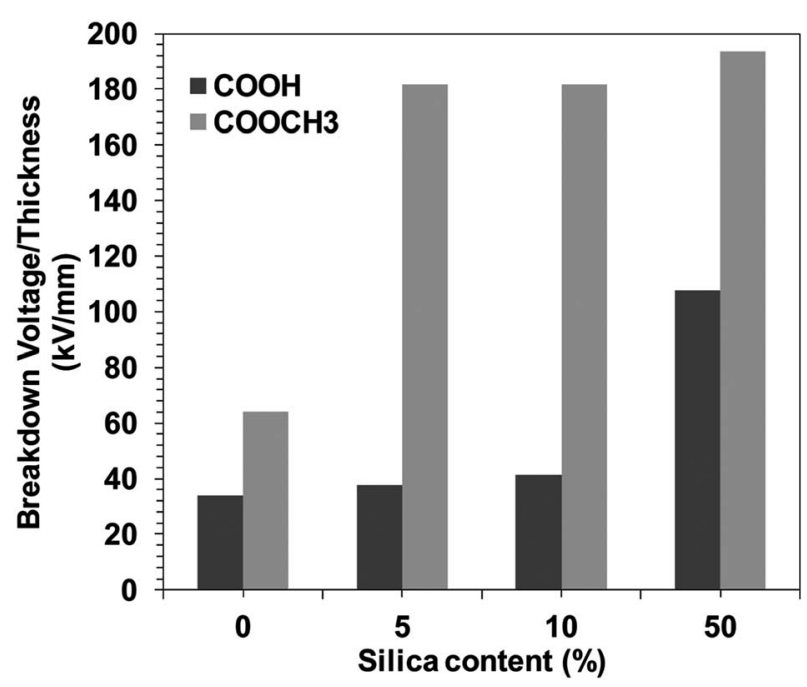

Fig. 3 Breakdown voltage of biopolyimide with various silica contents. 
(a)

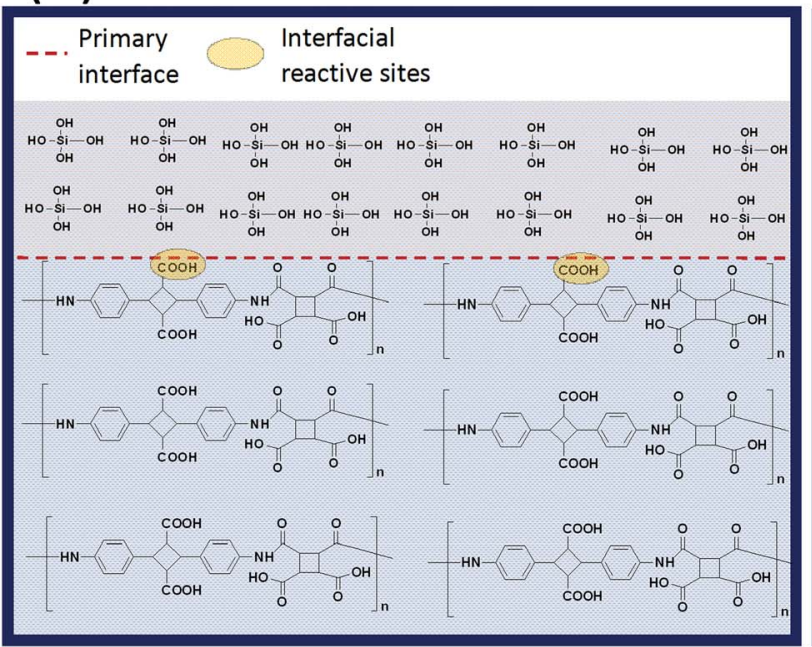

(c)

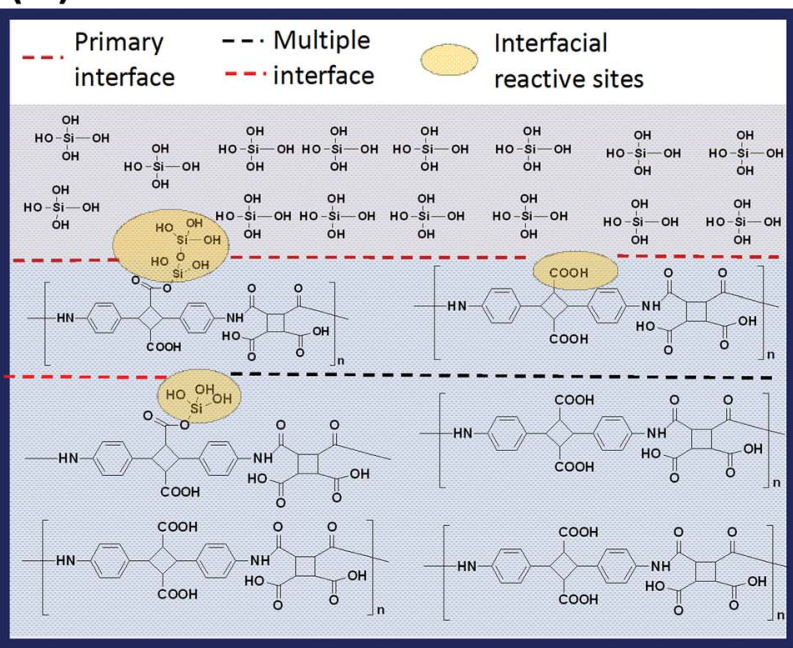

(e)

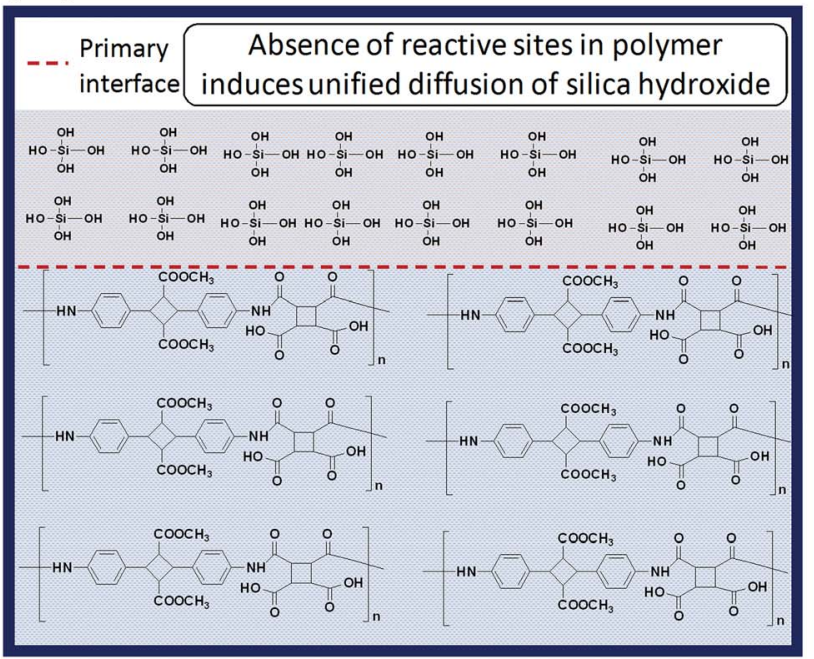

(b)

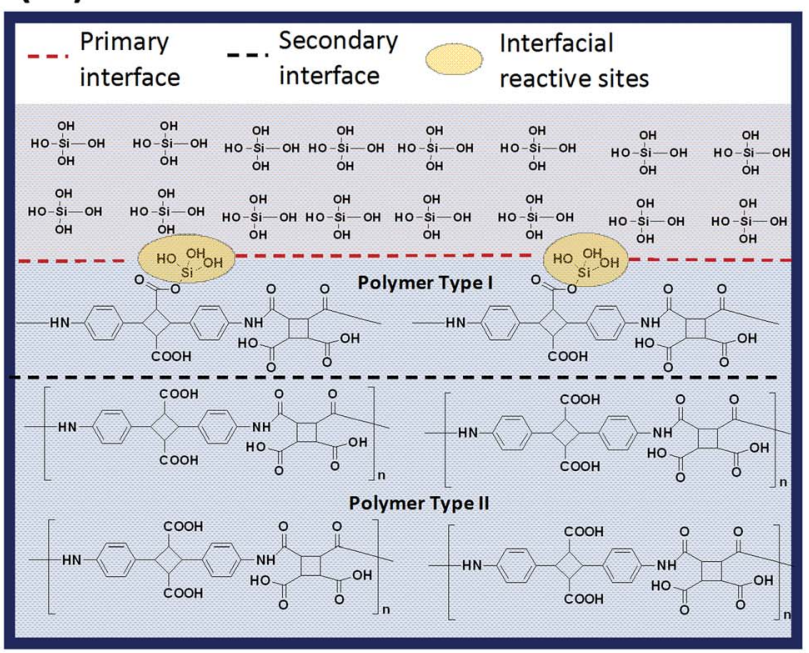

(d)

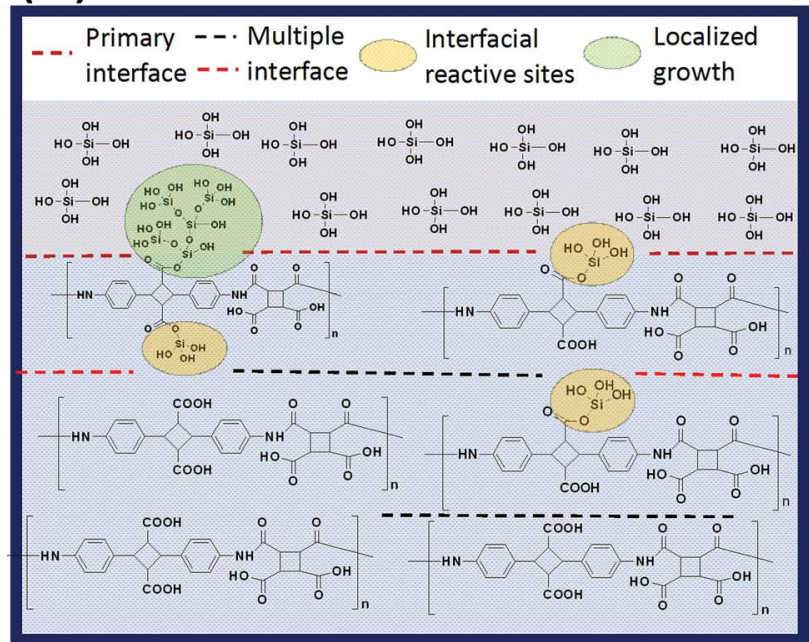

(f)

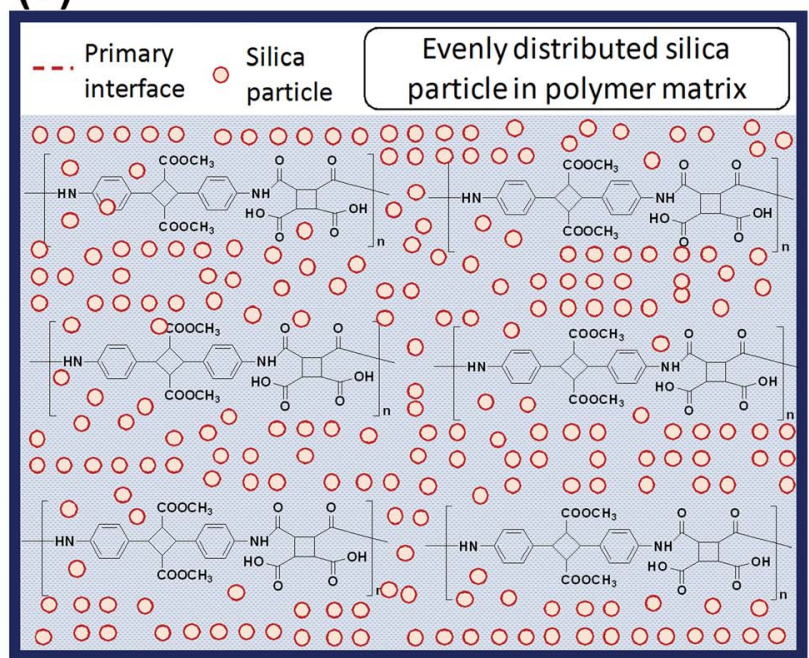

Scheme 2 Schematic illustration of silica particle growth during polymerization with different biopolyimide micro-structures. 
$\mathrm{kV} \mathrm{mm}^{-1}$ (neat BPI COOH $34 \mathrm{kV} \mathrm{mm}^{-1}$ ). On the contrary, in the case of $\mathrm{COOCH}_{3}$ type BPI, the DC breakdown voltage for the neat sample was $64 \mathrm{kV} \mathrm{mm}{ }^{-1}$ and increased up to more than $180 \mathrm{kV} \mathrm{mm}^{-1}$ at only $5 \mathrm{wt} \%$ silica concentration. This strongly indicates that the dispersion ability of silica within BPI matrix is different for $\mathrm{COOH}$ and $\mathrm{COOCH}_{3}$ type BPI. This behavior can be attributed to the depression in the percolation of electrical tree branching arose due to the uniform dispersion of the silica in the case of $\mathrm{COOCH}_{3}$ type BPI hybrids.

\section{Biopolyimide structure and silica hybrids performances}

Variation in the molecular structure may affect the resulting BPI (neat) silica hybrids properties, especially when one of the BPI contains a functionalization reactive towards the TEOS sol-gel condensation. The schematic illustration proposed for expressing the difference in the reaction environment for the sol-gel condensation is as represented in the Scheme 2. When, $\mathrm{COOH}$ type poly(amic acid) mixed with TEOS for the sol-gel condensation (Scheme 2(a)), two important aspects arises (1) interface between TEOS solution and the poly(amic acid) solution) and, (2) interfacial reactive sites 4ATA-COOH group (4,4'diamino- $\alpha$-truxillic acid moiety) reacts with the TEOS during sol-gel reaction. These two aspects greatly control the sol-gel reaction progress. As reaction proceeds (Scheme 2(b)), the hydrolysis of TEOS produces $\mathrm{Si}(\mathrm{OH})_{4}$ which possess a reactivity towards the reaction with the 4ATA-COOH groups along the interface and get linked with the main polymer chain. Moreover, the reaction of interfacial 4ATA-COOH groups and/or localized growth of the silanols lead to the formation of multiple interfaces in the reaction medium, which retards the diffusion of the TEOS $\left(\mathrm{Si}(\mathrm{OH})_{4}\right)$ in the reaction medium. Vigorous stirring of the reaction may lead to the forceful intermixing of the various interfaces and lead to the inter and/or intra-molecular linkage between the silanol molecules and the COOH type poly(amic acid) as shown in the (Scheme 2(c)). It is important to note that the interface shared between silanol molecules and over grown silanol-reacted $\mathrm{COOH}$ type poly(amic acid) grows much faster and lead to the non-uniform silica particle distribution along the polymer matrix (Scheme 2(d)) and reflected in the biopolyimide upon imidization. The nonuniform dispersion of the silica particle(s) in the polymer matrix lead to poor thermo-mechanical properties as well as opto-electrical properties. On the contrary, in the case of $\mathrm{COOCH}_{3}$ type poly(amic acid) the TEOS (or $\mathrm{Si}(\mathrm{OH})_{4}$ ) does not form multiple interfaces due to the absence of any reactive sites in the poly(amic acid) matrix. The absence of the reactive sites leads to a relatively more uniform diffusion of the silanol particles in the polymer matrix. The better dispersion of the silica particle in the polymer matrix leads to the superior thermo-mechanical, optical and electrical properties.

\section{Conclusions}

A new kind of biopolyimide has been synthesized and thereby a systematic study has been performed to understand the effect of polymer microstructure on their silica hybrids performances.
The hybrid films were obtained by the hydrolysispolycondensation of TEOS, by optionally facilitating reaction with the biopolyimide backbone functionalization. It is important to note that the introduction of silica in the poly(amic acid) backbone affects the properties of biopolyimide films. $\mathrm{COOCH}_{3}$ type BPI enables uniform dispersion of silica particle in the BPI matrix, which leads to improved thermo-mechanical, optical as well as ultra-high voltage endurance capabilities. On the other hand, the $\mathrm{COOH}$ containing BPI enabled the reaction of silanols with the carboxylic group. It has been proposed that the localized silanol reaction proceeds with non-uniform silica particle dispersion in the polymer matrix and therefore, possess properties lower than the matrix with uniform particle dispersion. Overall, the technique of controlling the chemical bond between inorganic/organic materials is an important and useful means to modulate the properties of hybrid materials. Summarizing, these finely tuned hybrid films are one of the most promising candidates for flexible transparent substrate especially for high temperature $\left(>350{ }^{\circ} \mathrm{C}\right)$ electronic and optoelectronic manufacturing processes.

\section{Conflicts of interest}

The authors declare no conflicts of interests.

\section{Acknowledgements}

The research was financially supported by Advanced Low Carbon Technology Research and Development Program (JST ALCA, 5100270), Tokyo, Japan.

\section{Notes and references}

1 H. H. Huang, B. Orler and G. L. Wilkes, Macromolecules, 1987, 20, 1322.

2 A. Morikawa, Y. Iyoku, M. Kakimoto and Y. Imai, Polym. J., 1992, 24, 107.

3 M. Nandi, J. A. Conklin, L. Salvati Jr and A. Sen, Chem. Mater., 1990, 2, 772.

4 Y. Watanabe, H. Sasabe, D. Yokoyama, T. Beppu, H. Katagiri and J. J. Kido, J. Mater. Chem. C, 2016, 4, 3699.

5 B. Deng, P. C. Hsu, G. Chen, B. N. Chandrashekar, L. Liao, Z. Ayitimuda, J. Wu, Y. Guo, L. Lin, Y. Zhou, M. Aisijiang, Q. Xie, Y. Cui, Z. Liu and H. Peng, Nano Lett., 2015, 15, 4206.

6 D. H. Kim, J. H. Ahn, W. M. Choi, H. S. Kim, T. H. Kim, J. Song, Y. Y. Huang, Z. Liu, C. Lu and J. A. Rogers, Science, 2008, 320, 507.

7 C. Wu, T. W. Kim, F. Li and T. Guo, ACS Nano, 2016, 10, 6449. 8 H. Lim, C. S. Cho, S. Ando, Y. K. Kim, C. H. Par and K. Lee, Adv. Mater., 2002, 14, 1275.

9 Y. Ji, B. Cho, S. Song, T. W. Kim, M. Choe, Y. H. Khang and T. Lee, Adv. Mater., 2010, 22, 3071.

10 D. J. Liaw, K. L. Wang, Y. C. Huang, K. R. Lee, J. Y. Lai and C. S. Ha, Prog. Polym. Sci., 2012, 37, 907.

11 W. C. Wang, R. H. Vora, E. T. Kang, K. G. Neoh, C. K. Ong and L. F. Chen, Adv. Mater., 2004, 16, 54. 
12 C. M. Leu, Z. W. Wu and K. H. Wei, Chem. Mater., 2002, 14, 3016.

13 W. H. Liao, S. Y. Yang, S. T. Hsiao, Y. S. Wang, S. M. Li and H. W. Tien, RSC Adv., 2014, 4, 51117.

14 W. Sun, J. Gu, L. Tian, P. Yao and Q. Zhang, J. Polym. Res., 2015, 22, 1.

15 E. Hamciuc, C. Hamciuc and M. Olariu, Polym. Eng. Sci., 2010, 50, 520.

16 T. Lee, S. S. Park, Y. Jung, S. Han, D. Han and I. Kim, Eur. Polym. J., 2009, 45, 19.

17 Y. J. Lee, J. M. Huang, S. W. Kuo, J. S. Lu and F. C. Chang, Polymer, 2005, 46, 173.

18 D. Li and Y. Xia, Adv. Mater., 2004, 16, 1151.

19 C. D. Saquing, J. L. Manasco and S. A. Khan, Small, 2009, 5, 944.

20 R. Vogelsang, T. Farr and K. Frohlich, IEEE Trans. Dielectr. Electr. Insul., 2006, 13, 373.

21 V. Tomer, G. Polizos, C. A. Randall and E. Manias, J. Appl. Phys., 2011, 109, 074113.

22 S. P. Fillery, H. Koerner, L. Drummy, E. Dunkerley, M. F. Durstock, D. F. Schmidt and R. A. Vaia, ACS Appl. Mater. Interfaces, 2012, 4, 1388.

23 V. Tomer, E. Manias and C. A. Randall, J. Appl. Phys., 2011, 110, 044107.

24 J. S. Meth, S. G. Zane, C. Z. Chi, J. D. Londono, B. A. Wood, P. Cotts, M. Keating, W. Guise and S. Weigand, Macromolecules, 2011, 44, 8301.

25 J. K. Nelson and J. C. Fothergill, Nanotechnology, 2004, 15, 586.

26 Z. M. Dang, Y. Q. Lin, H. P. Xu, C. Y. Shi and S. T. Li, Adv. Funct. Mater., 2008, 18, 1509.

27 T. T. Huang, C.-L. Tsai, S. Tateyama, T. Kaneko and G. S. Liou, Nanoscale, 2016, 8, 12793.

28 P. Kim, N. M. Doss, J. P. Tillotson, P. J. Hotchkiss, M. J. Pan, S. R. Marder, J. Li, J. P. Calame and J. W. Perry, ACS Nano, 2009, 3, 2581.

29 A. Morikawa, Y. Iyoku, M. Kakimoto and Y. Imai, J. Mater. Chem., 1992, 2, 679.

30 T. H. Lee, J. H. Kim and B. S. Bae, J. Mater. Chem., 2006, 16, 1657.

31 Y. S. Jung, S. J. Byun, S. J. Park and H. M. Lee, ACS Appl. Mater. Interfaces, 2014, 6, 6054.
32 P. Suvannasara, S. Tateyama, A. Miyasato, K. Matsumura, T. Shimoda, T. Ito, Y. Yamagata, T. Fujita, N. Takaya and

T. Kaneko, Macromolecules, 2014, 47, 1586.

33 S. Tateyama, S. Masuo, P. Suvannasara, Y. Oka, A. Miyasato,

K. Yasaki, T. Teerawatananond, N. Muangsin, S. Zhou,

Y. Kawasaki, L. Zhu, Z. Zhao, N. Takaya and T. Kaneko,

Macromolecules, 2016, 49, 3336.

34 Y. Cao and T. Mu, Ind. Eng. Chem. Res., 2014, 53, 8651.

35 L. L. Hench and J. K. West, Chem. Rev., 1990, 90, 33.

36 Z. Ahmad and J. Mark, Chem. Mater., 2001, 13, 3320.

37 Y. Chen and J. O. Iroh, Chem. Mater., 1999, 11, 1218.

38 B. K. Chen, T. M. Chiu and S. Y. Tsay, J. Appl. Polym. Sci., 2004, 94, 382.

39 W. D. Liu, B. K. Zhu, J. Zhang and Y. Y. Xu, Polym. Adv. Technol., 2007, 18, 522.

40 Y. Kim, E. Kang, Y. S. Kwon, W. J. Cho, C. Cho, M. Chang, M. Ree, T. Chang and C. S. Ha, Synth. Met., 1997, 85, 1399.

$41 \mathrm{~W} . \mathrm{Yu}, \mathrm{J} . \mathrm{Fu}, \mathrm{X}$. Dong, L. Chen and L. Shi, Compos. Sci. Technol., 2014, 92, 112.

42 J. Liu, Y. Min, J. Chen, H. Zhou and C. Wang, Macromol. Rapid Commun., 2007, 28, 215.

43 Y. Li, X. Lu, C. Zhang, X. Li and W. Zhang, Appl. Phys. A, 2010, 100, 207.

44 K. Taki, K. Hosokawa, S. Takagi, H. Mabuchi and M. Ohshima, Macromolecules, 2013, 46, 2275.

45 L. B. Nichols and J. M. Thorp, J. Chem. Soc., Faraday Trans., 1970, 66, 1741.

46 S. Ducharme, ACS Nano, 2009, 3, 2447.

47 L. A. Fredin, Z. Li, M. A. Ratner, M. T. Lanagan and T. J. Marks, Adv. Mater., 2012, 24, 5946.

48 H. Scher and R. J. Zallen, Chem. Phys., 1970, 53, 3759.

49 K. Wu, L. A. Dissado and T. Okamoto, Appl. Phys. Lett., 2004, 85, 4454.

50 S. Siddabattuni, T. P. Schuman and F. Dogan, Mater. Sci. Eng., B, 2011, 176, 1422.

51 M. Roy, J. K. Nelson, R. K. MacCrone and L. S. Schadler, J. Mater. Sci., 2007, 42, 3789.

52 S. J. Laihonen, U. Gafvert, T. Schutte and U. W. Gedde, IEEE Trans. Dielectr. Electr. Insul., 2007, 14, 275.

53 J. Brandup, E. H. Immergut, E. A. Grulke and D. Bloch, Polymer Handbook, edn 4th, Wiley, New York, 1999. 